\title{
Effective Velocity of Soliton in the Presence of a Periodic Background
}

\author{
J. PEŁKA* AND J. ZAGROdZIŃSKI \\ Institute of Physics, Polish Academy of Sciences \\ al. Lotników 32/46, 02-668 Warsaw, Poland
}

(Received September 3, 2001)

\begin{abstract}
The effective soliton velocity in the presence of a periodic background differs from that when a soliton propagates alone. Using approximate relations for the Riemann-theta functions, the effective soliton velocity is derived and discussed. General relations are illustrated by examples of Korteweg de Vries and sine-Gordon equations because of the application in the Josephson junction theory.
\end{abstract}

PACS numbers: 42.50.Md, 42.81.Dp, 74.50.+r, 85.25.Cp

\section{Introduction}

There exists a lot of physical problems which can be modeled by the soliton propagation on a periodic background. In such a situation solitons play a role of localized excitements and periodic phases mimic a phonon, lattice or magnetic field background. As a rule, the background of this type is periodic or quasi-periodic. This problem has a long tradition, starting from the Rubinstein paper [1], where the perturbative method for the sine-Gordon $(\mathrm{sG}$ ) partial differential equation was considered in order to determine a mutual interaction. The fluxon propagation in the ring Josephson junction in the presence of defects $[2,3]$ or in the junction polarized either by the dc current or dc external magnetic field [4] make another examples. The lattice of defects in the first example or dc background in the second one can be considered as a periodic phase and the propagation of distinguished excitements is modified by the presence of these backgrounds. The problem of

*corresponding author; e-mail: pelkay@ifpan.edu.pl 
finding this effective velocity appears also in other physical situations, where localized excitations appear together with delocalized background perturbation and the principle of superposition cannot be applied.

\section{2. "Mixed" solution of $\mathrm{sG}$ equation}

As it is commonly known from the soliton theory, due to the interaction of a localized soliton with a periodic process, only the phase shift appears (see Fig. 1). The same conclusion relates to an interaction with periodic process since the simplest periodic process emerging from sG equation ( $\mathrm{sGe}$ ) formally can be considered as a sum of an infinite number of solitons shifted equidistantly in space [5]. The relevant relation has the following form:

$$
u(x, t)=\sum_{n=1}^{\infty} 2 \mathrm{i} \ln \left(\frac{1-\mathrm{i} \exp a_{n}}{1+\mathrm{i} \exp a_{n}}\right)+\sum_{n=0}^{-\infty} 2 \mathrm{i} \ln \left(\frac{\mathrm{i} \exp a_{n}-1}{\mathrm{i} \exp a_{n}+1}\right),
$$

where $a_{n}=k x-\omega t+2 \pi \mathrm{i} n b$, and the $b$ constant is roughly speaking proportional to its period. (Unfortunately this interpretation is valid only for a single phase periodic solution.)

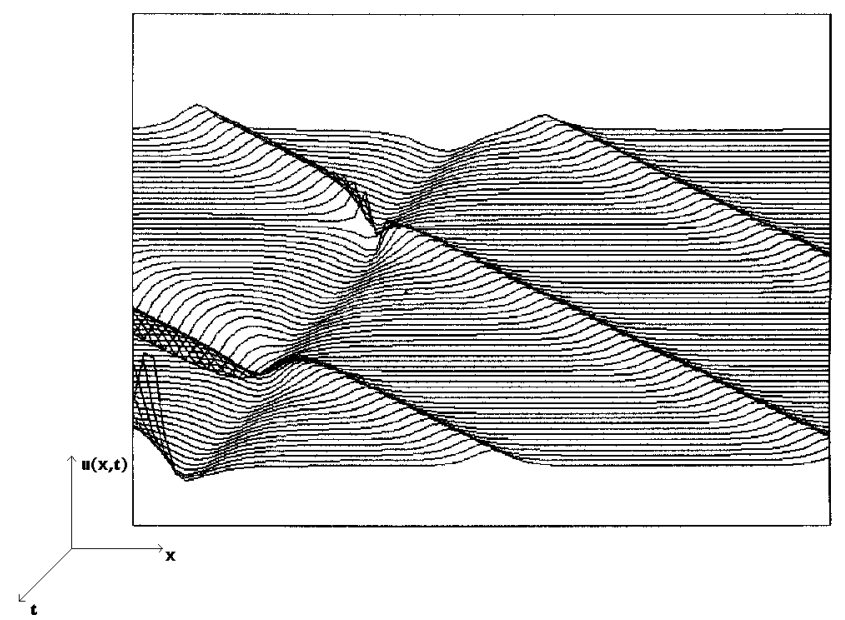

Fig. 1. The 3-phases solution of sine-Gordon equation, i.e. one antisoliton interacts with the quasi-periodic phase. Its effective velocity has the form of Eq. (10).

As a consequence of the periodically repeated phase shifts, the velocity of the soliton is changed. This means that its velocity (which is equal to $\omega / \kappa$, when the soliton propagates alone), due to the series of phase shifts induced by a periodic process, is changed and the soliton travels with another velocity which we denote as the "effective velocity".

We relate these general statements to the $\mathrm{sG}$ equation, just because of the Rubinstein paper, although all considerations are valid also for the other 
soliton-type equations. The formalism bases on the extraction of the soliton phase from a solution which represents a multiphase quasiperiodic process.

The first attempt to this problem one can find in the above-mentioned Rubinstein paper [1]. Since at that time (1982) an exact solution was unknown, the author analyzed the interaction of the soliton with a small, perturbative periodic process and found a phase shift and dispersion parameters of the localized single kink solution of the sine-Gordon equation.

In contrast to this approach we consider the exact, mixed solution of sine-Gordon equation [6]. As a starting point, the solution, in a form of the kink on a periodic background (of sG equation), can be written as

$$
u(x, t)=2 \mathrm{i} \ln \frac{R^{*}(z, B)}{R(z, B)},
$$

where $R(z, B)=\Theta\left(z_{\mathrm{p}} \mid B_{\mathrm{pp}}\right)+\exp \left(12 \pi z_{\mathrm{s}}\right) \Theta\left(z_{\mathrm{p}}+B_{\mathrm{sp}} \mid B_{\mathrm{pp}}\right), \quad z=\left(z_{\mathrm{s}}, z_{\mathrm{p}}\right), \quad z_{\mathrm{s}}=$ $k_{\mathrm{s}} x+\omega_{\mathrm{s}} t+z_{0 \mathrm{~s}}, z_{\mathrm{p}}=k_{\mathrm{p}} x+\omega_{\mathrm{p}} t+z_{0 \mathrm{p}}, \quad z_{0 \mathrm{~s}}$ and $z_{0 \mathrm{p}}$ are arbitrary constants. Indices $\mathrm{s}$ and $\mathrm{p}$ refer to soliton and periodic processes, respectively. $\Theta$ is the Riemann-theta function (or Jacobi $\vartheta_{3}$ function) of the argument $z_{\mathrm{p}}$ and parameter $B_{\mathrm{pp}}[7]$

$$
\Theta\left(z_{\mathrm{p}} \mid B_{\mathrm{pp}}\right)=\sum_{n=-\infty}^{\infty} \exp \left[\mathrm{i} \pi\left(2 z_{\mathrm{p}} n+B_{\mathrm{pp}} n^{2}\right)\right]
$$

The propagation constants $k_{\mathrm{s}}, \mathrm{k}_{\mathrm{p}}$ and angular velocities $\omega_{\mathrm{s}}, \omega_{\mathrm{p}}$ of the soliton and periodic phases are determined by a system of relevant algebraic dispersion equations [8]. In the case of the two-phase solution of $\mathrm{sG}$ equation it has the following form of a four-equation system:

$$
\sum_{i, j=1}^{2}\left(k_{i} k_{j}-\omega_{i} \omega_{j}\right) \frac{\partial}{\partial w_{i} \partial w_{j}} W_{\varepsilon}(w)_{w=0}+\frac{1}{4}\left(\delta_{\varepsilon, e}-\delta_{\varepsilon, 0}\right)=0
$$

for $\varepsilon=(0,0),(1,0),(0,1),(1,1)$, where $e=(1,1), \delta_{\varepsilon, e}$ is the Kronecker symbol and $W_{\varepsilon}(w)$ are the known coefficients [9]. As the first step, one obtains parameters of the periodic process and these are independent of the existence of soliton phase. In contrast, the soliton parameters depend on parameters of periodic phase. From the dispersion equations (4) it is seen that the periodic process changes the parameters $\left(k_{\mathrm{s}}, \omega_{\mathrm{s}}\right)$ of the coexisting soliton process.

Relation (2) can be derived as a limiting procedure applied with respect to the two-phase quasi-periodic solution when the imaginary part of some $B$-matrix diagonal element tends to infinity

$$
R(z, B)_{(\mathrm{s}, \mathrm{p})}=\lim _{D^{\mathrm{ss}} \rightarrow \infty} \Theta\left(\left[\begin{array}{c}
z^{\mathrm{s}}-\frac{\mathrm{i}}{2} D^{\mathrm{ss}} d^{\mathrm{s}} \\
z^{\mathrm{p}}
\end{array}\right] \mid\left[\begin{array}{cc}
\mathrm{i} D^{\mathrm{ss}}+\tilde{B}^{\mathrm{ss}} & B^{\mathrm{sp}} \\
B^{\mathrm{ps}} & B^{\mathrm{pp}}
\end{array}\right]\right)
$$

where $D^{\text {ss }}:=\operatorname{diag}\left(\operatorname{Im} B^{\text {ss }}\right)$. When the trick $(5)$ is repeated with respect to both phases, $R(z, B)=1+\mathrm{i}\left(\exp \zeta_{1}+\exp \zeta_{2}\right)-\exp \left(\zeta_{1}+\zeta_{2}+\beta_{12}\right)$ and we arrive at the famous double-soliton formula [10]. 
In our case the mixed exact solution of $\mathrm{sG}$ equation has the following form:

$$
u(z, B)=2 \mathrm{i} \ln \frac{\left[\Theta\left(z_{\mathrm{p}} \mid B_{\mathrm{pp}}\right)+\exp \left(\mathrm{i} 2 \pi z_{\mathrm{s}}\right) \Theta\left(z_{\mathrm{p}}+B_{\mathrm{sp}} \mid B_{\mathrm{pp}}\right)\right]^{*}}{\Theta\left(z_{\mathrm{p}} \mid B_{\mathrm{pp}}\right)+\exp \left(\mathrm{i} 2 \pi z_{\mathrm{s}}\right) \Theta\left(z_{\mathrm{p}}+B_{\mathrm{sp}} \mid B_{\mathrm{pp}}\right)} .
$$

When we are looking for a soliton propagating on the background of an oscillatory periodic process, the requirements of the real solutions of sGe are as follows: $z_{\mathrm{s}}=1 / 4+\mathrm{i} \eta, z_{\mathrm{p}}=\mathrm{i} \xi$ and $B_{\mathrm{sp}}=-\mathrm{i} b, B_{\mathrm{pp}}=1 / 2+\mathrm{i} \beta$, where $\eta, \xi, b$, and $\beta$ are real. We neglect initial positions given by $z_{0 \mathrm{~s}}$ and $z_{0 \mathrm{p}}$.

It is important to note that the obviously known relation $\omega^{2}-k^{2}=1$ is true only for "pure" soliton solutions of sG equation. In our mixed case of course $\omega^{2}-k^{2} \neq 1$

\section{Effective velocity for a mixed solution of sGe}

The approximate formula for the first order $\Theta$ function (Jacobi function) valid for our approach when $\beta \ll 1$ is

$$
\begin{aligned}
& \Theta\left(\eta+\mathrm{i} \xi \mid \frac{\tau}{2}+\mathrm{i} \beta\right) \\
& \cong \frac{1}{2 \sqrt{\beta}} \exp \left(-\mathrm{i} \pi \frac{2 \eta \xi}{\beta}\right) \exp \left[\frac{\pi}{\beta}\left(\xi^{2}-\eta^{2}\right)\right]\left[1+\exp \left(\mathrm{i} \pi \frac{\tau}{2}\right)\right],
\end{aligned}
$$

which when $\eta=\tau=0$ reduces to the commonly known relation [11]

Substituting (7) into (6), after simple manipulations, our mixed solution of sGe in case of a single soliton interacting with a (single) periodic (oscillatory) phase has the approximate form

$$
\begin{gathered}
u(\xi, \eta, b, \beta)=\pi+2 \mathrm{i} \ln \frac{1-\mathrm{i} \exp \left[-2 \pi(\beta \eta+b \xi)-\pi b^{2}\right]}{1+\mathrm{i} \exp \left[-2 \pi(\beta \eta+b \xi)-\pi b^{2}\right]} \\
=\pi+4 \arctan \left\{\exp \left[-2 \pi(\beta \eta+b \xi)-\pi b^{2}\right]\right\} .
\end{gathered}
$$

It is seen that it represents a single soliton solution of sGe, but with a new velocity and shifted by $-b^{2} / 2 \beta$. Since arguments are linear in the space and time coordinates $\xi=k_{\mathrm{p}} x+\omega_{\mathrm{p}} t, \eta=k_{\mathrm{s}} x+\omega_{\mathrm{s}} t$, then

$$
\beta \eta+b \xi=x\left(\beta k_{\mathrm{s}}+b k_{\mathrm{p}}\right)+t\left(\beta \omega_{\mathrm{s}}+b \omega_{\mathrm{p}}\right),
$$

which means that the effective velocity $v_{\mathrm{eff}}$ of this soliton is

$$
v_{\mathrm{eff}}=\frac{\omega_{\mathrm{eff}}}{k_{\mathrm{eff}}}=\frac{\beta \omega_{\mathrm{s}}+b \omega_{\mathrm{p}}}{\beta k_{\mathrm{s}}+b k_{\mathrm{p}}},
$$

where $\omega_{\mathrm{s}}, k_{\mathrm{s}}$ are ascribed to the soliton process and $\omega_{\mathrm{p}}, k_{\mathrm{p}}$ - to the periodic one.

It is seen that this velocity differs from the ("phase") soliton velocity $v_{\mathrm{s}}=\omega_{\mathrm{s}} / k_{\mathrm{s}}$. Since $b$ stands for the coupling between a soliton and periodic processes, the stronger coupling - the bigger changes $k_{\text {eff }}$ and $\omega_{\text {eff }}$. On the other side, $\beta$ is roughly speaking proportional to the period of periodic process. Then when this period is bigger, $v_{\mathrm{eff}}$ is closer to $v_{\mathrm{s}}$. 
Although the dispersion relations give the expression $f\left(b, k_{\mathrm{p}}, \omega_{\mathrm{p}}\right)=0$, but unfortunately also $k_{\mathrm{s}}=k_{\mathrm{s}}\left(b, \beta, k_{\mathrm{p}}, \omega_{\mathrm{p}}\right)$ and $\omega_{\mathrm{s}}=\omega_{\mathrm{s}}\left(b, \beta, k_{\mathrm{p}}, \omega_{\mathrm{p}}\right)$ and thus the change of $\beta$ in (10) gives rise to changes of $k_{\mathrm{s}}$ and $\omega_{\mathrm{s}}$ too, and only $k_{\mathrm{p}}$ and $\omega_{\mathrm{p}}$ remain unchanged. Therefore (10) have to be read as $v_{\text {eff }}=\left[\beta \omega_{\mathrm{s}}\left(b, \beta, k_{\mathrm{p}}, \omega_{\mathrm{p}}\right)+b \omega_{\mathrm{p}}\right] /$ $\left[\beta k_{\mathrm{s}}\left(b, \beta, k_{\mathrm{p}}, \omega_{\mathrm{p}}\right)+b k_{\mathrm{p}}\right]$.

Similar relations can be achieved for soliton propagating on the background of rotational periodic process where the phase is growing monotonically in time. Equation (6) is still valid, but now $z_{\mathrm{s}}=1 / 4+\mathrm{i} \eta, z_{\mathrm{p}}=1 / 4+\mathrm{i} \xi$ and $B_{\mathrm{sp}}=-\mathrm{i} b$, $B_{\mathrm{pp}}=\mathrm{i} \beta$, where $\eta, \xi, b$, and $\beta$ are real. Repeating the procedure as above, we have

$$
u(\xi, \eta, b, \beta)=-2 \pi \frac{\xi}{\beta}+2 \mathrm{i} \ln \frac{1-\mathrm{i} \exp \left[-2 \pi\left(\beta \eta_{\mathrm{s}}+b \xi-s\right)^{*}\right]}{1+\mathrm{i} \exp \left[-2 \pi\left(\beta \eta_{\mathrm{s}}+b \xi-s\right)\right]}
$$

This represents, once more a single soliton solution but with a term linearly growing in time (or space) $-2 \pi \xi / \beta$, shifted by $s=(b / 2)(b-\mathrm{i} / 2)$. Note that besides the complex shift the whole approximate single soliton solution remains real. The effective velocity is given by (10), as before.

\section{Effective velocity for mixed solution of Korteweg de Vries}

Another example, even simpler than sGe, is the Korteweg de Vries (KdV) equation. Starting from the mixed solution of $\mathrm{KdV}$ equation, we obtain a similar single soliton-like solution with a constant background

$$
\begin{aligned}
& u(x, t)=\left\{\ln \left[\Theta\left(z_{\mathrm{p}} \mid B_{\mathrm{pp}}\right)+\exp \left[\mathrm{i} \pi\left(2 z_{\mathrm{s}}\right)\right] \Theta\left(z_{\mathrm{p}}+B_{\mathrm{sp}} \mid B_{\mathrm{pp}}\right)\right]\right\}_{x x} \\
& =\frac{\pi \sqrt{\beta}}{\beta^{2}} k_{\mathrm{p}}{ }^{2}+\left\{\ln \left[1+\exp \left(2 \pi\left[\left(\beta k_{\mathrm{s}}+b k_{\mathrm{p}}\right) x+\left(\beta \omega_{\mathrm{s}}+b \omega_{\mathrm{p}}\right) t-b^{2} / 2\right]\right)\right]\right\}_{x x}
\end{aligned}
$$

with the meaning of symbols and all comments as before.

The same approach can be applied to a single soliton process on the two-phase quasi-periodic background. For simplicity we confine ourselves to the KdV equation.

The starting solution in this case has the form

$$
\begin{aligned}
& u(x, t)=\left\{\ln \Theta\left(\left[\begin{array}{l}
z_{1} \\
z_{2}
\end{array}\right] \mid\left[\begin{array}{ll}
B_{11} & B_{12} \\
B_{12} & B_{22}
\end{array}\right]\right)\right. \\
& \left.+\exp \left(\mathrm{i} 2 \pi z_{3}\right) \Theta\left(\left[\begin{array}{l}
z_{1}+B_{13} \\
z_{2}+B_{23}
\end{array}\right] \mid\left[\begin{array}{ll}
B_{11} & B_{12} \\
B_{12} & B_{22}
\end{array}\right]\right)\right\}_{x x},
\end{aligned}
$$

with $z_{j}=\mathrm{i} \xi_{j}, B_{j k}=\mathrm{i} \beta_{j k},\left(\xi_{j}, \beta_{j k}\right) \in R, i, j=1,2,3$. Indices 1,2 relate to periodic phases and index 3 to the soliton one. 
Using the approximate relation for the second order $\Theta$ function with the imaginary matrix $B$ and arguments, similarly as before, in case of the Jacobi functions (7), we obtain

$$
\begin{aligned}
& \Theta\left(\left[\begin{array}{l}
\mathrm{i} \xi_{1} \\
\mathrm{i} \xi_{2}
\end{array}\right] \mid\left[\begin{array}{ll}
\mathrm{i} \beta_{11} & \mathrm{i} \beta_{12} \\
\mathrm{i} \beta_{12} & \mathrm{i} \beta_{22}
\end{array}\right]\right) \\
\cong & \int_{-\infty}^{\infty} \int_{-\infty}^{\infty} \exp \left[-\pi\left(2 \xi_{1} n+2 \xi_{2} m+\beta_{11} n^{2}+2 \beta_{12} n m+\beta_{22} m^{2}\right)\right] \mathrm{d} n \mathrm{~d} m \\
= & \frac{1}{\sqrt{\beta_{11} \beta_{22}-\beta_{12}^{2}}} \exp \left[\frac{\pi\left(\beta_{11} \xi_{2}^{2}+\beta_{22} \xi_{1}^{2}-2 \beta_{12} \xi_{1} \xi_{2}\right)}{\beta_{11} \beta_{22}-\beta_{12}^{2}}\right]
\end{aligned}
$$

for $\beta_{11} \beta_{22}-\beta_{12}^{2} \ll 1$, and next

$$
\begin{gathered}
u(x, t)=\left\{\operatorname { l n } \left[\frac{1}{\sqrt{\beta_{11} \beta_{22}-\beta_{12}^{2}}} \exp \frac{\pi\left(\beta_{11} \xi_{2}^{2}+\beta_{22} \xi_{1}^{2}-2 \beta_{12} \xi_{1} \xi_{2}\right)}{\beta_{11} \beta_{22}-\beta_{12}^{2}}\right.\right. \\
+\exp \left(-2 \pi \xi_{3}\right) \frac{1}{\sqrt{\beta_{11} \beta_{22}-\beta_{12}^{2}}} \\
\left.\left.\times \exp \frac{\pi\left[\beta_{11}\left(\xi_{2}-\beta_{23}\right)^{2}+\beta_{22}\left(\xi_{1}-\beta_{13}\right)^{2}-2 \beta_{12}\left(\xi_{1}-\beta_{13}\right)\left(\xi_{2}-\beta_{23}\right)\right]}{\beta_{11} \beta_{22}-\beta_{12}^{2}}\right]\right\}_{x x}
\end{gathered}
$$

Another version of an approximate relation was reported in [12].

Since $\xi_{j}=k_{j} x+\omega_{j} t, j=1,2,3$, then

$$
u=\Gamma+[\ln (1+\exp \gamma)]_{x x},
$$

where

$$
\begin{aligned}
& \Gamma=2 \pi\left(\beta_{11} k_{2}+\beta_{22} k_{1}-2 \beta_{12} k_{2} k_{1}\right) / \Delta, \quad \Delta=\beta_{11} \beta_{22}-\beta_{12}^{2} \\
& \gamma=-2 \pi\left[\left(p k_{2}+q k_{1}+k_{3}\right) x+\left(p \omega_{2}+q \omega_{1}+\omega_{3}\right) t+s\right]
\end{aligned}
$$

and

$$
\begin{aligned}
& p=\left(\beta_{11} \beta_{23}-\beta_{12} \beta_{13}\right) / \Delta, \quad q=\left(\beta_{13} \beta_{22}-\beta_{12} \beta_{23}\right) / \Delta, \\
& s=\left(2 \beta_{12} \beta_{13} \beta_{23}-\beta_{11} \beta_{23}^{2}-\beta_{22} \beta_{13}^{2}\right) / 2 \Delta .
\end{aligned}
$$




\section{Conclusions}

It is seen once more that the expression (16) represents a single soliton solution with modified $k_{\text {eff }}$ and $\omega_{\text {eff }}$. Thus, the effective velocity of a soliton on the two-phase periodic background is equal to

$$
v_{\mathrm{eff}}=\frac{\omega_{\mathrm{eff}}}{k_{\mathrm{eff}}}=\frac{\omega_{3}+\left(q \omega_{1}+p \omega_{2}\right)}{k_{3}+\left(q k_{1}+p k_{2}\right)}
$$

where the terms in parenthesis appeared due to the interaction with the background. One can discuss of course whether and when this contribution is small or large.

The analogous approach can be applied to other soliton-type equations.

\section{Acknowledgments}

The authors acknowledge the support of the State Committee for Scientific Research (KBN), grant No. 2P03B-114-11.

\section{References}

[1] J. Rubinstein, J. Math. Phys. 11, 258 (1970).

[2] A.V. Ustinov, T. Doderer, R.P. Huebener, N.F. Pedersen, B. Mayer, V.A. Oboznov, Phys. Rev. Lett. 69, 1815 (1992).

[3] A. Shnirman, Z. Hermon, A.V. Ustinov, B.A. Malomed, A. Ben-Jacob, Phys. Rev. B 50, 793 (1994).

[4] J. Pełka, J. Zagrodziński, Physica B 154, 125 (1989).

[5] M.G. Forest, D.W. McLaughlin, J. Math. Phys, 23, 1248 (1982).

[6] J. Zagrodziński, M. Jaworski, J. Phys. B, Condens. Matter 49, 75 (1982).

[7] D. Mumford, Tata Lectures on Theta Functions I, Birkhauser, Boston 1983; Tata Lectures on Theta Functions II, Birkhauser, Boston 1984.

[8] J. Zagrodziński, Phys. Rev. B 53, 59 (1996).

[9] J. Zagrodziński, J. Phys. A, Math. Gen. 15, 3109 (1982).

[10] M.J. Ablowitz, H. Segur, Solitons and the Inverse Scattering Transform, SIAM, Philadelphia 1981.

[11] F. Oberhettinger, W. Magnus, Adwendung der Elliptischen Functionen in Physik und Technik, Springer, Berlin 1949.

[12] M. Jaworski, Phys. Lett. A 244, 97 (1998). 